This is the final peer-reviewed accepted manuscript of:

Borhani, K., Borgomaneri, S., Làdavas, E., \& Bertini, C. (2016). The effect of alexithymia on early visual processing of emotional body postures. Biological Psychology, 115, 18.

The final published version is available online at:

https://doi.org/10.1016/j.biopsycho.2015.12.010

Rights / License:

The terms and conditions for the reuse of this version of the manuscript are specified in the publishing policy. For all terms of use and more information see the publisher's website.

This item was downloaded from IRIS Università di Bologna (https://cris.unibo.it/)

When citing, please refer to the published version. 


\section{The effect of alexithymia on early visual processing of emotional body}

\section{postures}

Khatereh Borhani $^{\mathrm{a}, \mathrm{b}}$, Sara Borgomaneri ${ }^{\mathrm{a}, \mathrm{b}}$, Elisabetta Làdavas ${ }^{\mathrm{a}, \mathrm{b}}$, Caterina Bertini ${ }^{\mathrm{a}, \mathrm{b}^{*}}$

${ }^{a}$ Department of Psychology, University of Bologna, Viale Berti Pichat 5, 40127, Bologna, Italy;

${ }^{\mathrm{b}}$ CSRNC, Centre for Studies and Research in Cognitive Neuroscience, University of Bologna, Viale Europa 980, 47521, Cesena, Italy.

Khatereh Borhani: khatereh.borhani3@unibo.it

Sara Borgomaneri: sara.borgomaneri2@unibo.it

Elisabetta Làdavas: elisabetta.ladavas@unibo.it

Caterina Bertini: caterina.bertini@unibo.it

*Corresponding Author:

Caterina Bertini

Department of Psychology, University of Bologna

Viale Berti Pichat 5, 40127, Bologna, Italy

Phone +390512091347

Fax: +390512091844

E-mail: caterina.bertini@unibo.it 


\section{Abstract}

Body postures convey emotion and motion-related information useful in social interactions. Early visual encoding of body postures, reflected by the N190 component, is modulated both by motion (i.e., postures implying motion elicit greater N190 amplitudes than static postures) and by emotionrelated content (i.e., fearful postures elicit the largest N190 amplitude). At a later stage, there is a fear-related increase in attention, reflected by an early posterior negativity (EPN) (Borhani et al., 2015). Here, we tested whether difficulties in emotional processing (i.e., alexithymia) affect early and late visual processing of body postures. Low alexithymic participants showed emotional modulation of the N190, with fearful postures specifically enhancing N190 amplitude. In contrast, high alexithymic participants showed no emotional modulation of the N190. Both groups showed preserved encoding of the motion content. At a later stage, a fear-related modulation of the EPN was found for both groups, suggesting that selective attention to salient stimuli is the same in both low and high alexithymia.

Keywords: emotional bodies; alexithymia; visual structural encoding; N190; Early Posterior Negativity (EPN). 


\section{Introduction}

The ability to perceive and categorize emotional stimuli is highly relevant in social environments. Indeed, rapid processing of potentially threatening stimuli is crucial for minimizing the negative consequences associated with unpleasant cues. In support of this view, recent evidence has shown that unpleasant stimuli are detected more quickly than both pleasant and neutral stimuli (Fox et al., 2000; Hansen and Hansen, 1988; Öhman et al., 2001). In addition, negative stimuli are associated with enhanced activation in perceptual occipito-temporal areas (Taylor et al., 2000) and in subcortical structures, such as the amygdala, that are pivotal for emotional processing (Breiter et al., 1996; Fusar-Poli et al., 2009; Lane et al., 1998; Oya et al., 2002). These findings suggest that more processing resources are devoted to the visual processing of unpleasant stimuli than to pleasant or neutral stimuli (Carretié et al., 2009; Vuilleumier, 2002). Electrophysiological studies have also shown that fearful faces enhance early event-related potential (ERP) components such as the P1, reflecting exogenous spatial orienting of attention toward fearful stimuli (Pourtois et al., 2005, 2004). In addition, both explicit (Batty and Taylor, 2003; Stekelenburg and de Gelder, 2004) and implicit (Cecere et al., 2014; Pegna et al., 2011, 2008) processing of fearful faces can modulate early stages of perceptual encoding of facial features and configurations, as indexed by the occipitotemporal N170 component (Batty and Taylor, 2003; Bentin et al., 1996). Moreover, at a later stage of perceptual representation (around $300 \mathrm{~ms}$ after stimulus onset), faces expressing negative emotions increase stimulus-driven attentional capture, as suggested by a pronounced early posterior negativity (EPN; Bayer and Schacht, 2014; Calvo and Beltrán, 2014; Frühholz et al., 2011; Rellecke et al., 2012; Schupp et al., 2004; Valdés-Conroy et al., 2014).

Besides facial expressions, human body postures represent a powerful tool for inferring the internal states of others (de Gelder et al., 2010). Indeed, body postures convey information about others' actions and emotions, both of which are useful for interpreting goals, intentions and mental states. Compared to faces, body postures offer the possibility to capture these signals from longer 
distances. Similar to the face-related N170, the observation of bodies elicits an early occipitotemporal negative deflection peaking at $190 \mathrm{~ms}$ after stimulus presentation, which has been termed the N190. It is thought to be generated in a restricted area of the occipito-temporal cortex, corresponding to the extrastriate body area (EBA; Meeren et al., 2013; Taylor et al., 2010; Thierry et al., 2006). This electrophysiological signature reflects the extraction of abstract properties of the human body form for categorization (Thierry et al., 2006), and represents the earliest component indexing structural features of human bodies (Taylor et al., 2010). Interestingly, both motion- and emotion-related information conveyed by body postures can modulate the N190 (Borhani et al., 2015). Indeed, in a recent electrophysiological study (Borhani et al., 2015), bodies with static or implied motion postures, and with or without emotional content (fearful, happy or neutral), were presented peripherally to the left or the right of a central fixation point. The N190 component, recorded from the right hemisphere, was modulated both by the presence of implied motion (i.e., larger N190 amplitude in response to body postures conveying implied motion compared to static postures) and by emotional content (i.e., larger N190 amplitude in response to fearful body postures). These modulations suggest that this visual processing stage encodes not only a perceptual representation of the visual stimulus as a body, but also a more detailed analysis of motion and emotion information conveyed by body postures.

Notably, the study by Borhani et al. (2015) did not show any modulation of the early P1 component. This is in keeping with other electrophysiological findings (Stekelenburg and de Gelder, 2004) that emotional stimuli modulate ERP components in the same time window as the N190 (i.e., the Vertex Positive Potential, which is considered the fronto-central counterpart of the N190), but not earlier components. Moreover, evidence for modulations of the P1 in response to emotional bodies is inconsistent (Meeren et al., 2005; van Heijnsbergen et al., 2007), possibly because the P1 is highly sensitive to the physical properties of the stimulus (Halgren et al., 2000; Rossion and Jacques, 2008). 
At a later stage of perceptual representation (i.e., $300 \mathrm{~ms}$ post-stimulus onset), viewing fearful body postures elicits a pronounced early posterior negativity (EPN; Borhani et al., 2015). The EPN is a ERP difference in the processing of emotionally relevant stimuli and neutral stimuli, and occurs 200-300 ms after stimulus presentation (Schupp et al., 2006). This differential ERP appears as a negative deflection over temporo-parietal areas, and reflects exogenous attentional capture driven by salient emotional stimuli and the degree of attention needed to recognize relevant signals (Olofsson et al., 2008), such as body postures expressing fear. These results suggest the existence of a specialized perceptual mechanism tuned to the emotion and action-related information conveyed by human body postures.

Recent evidence suggests that individual emotional skills (Meaux et al., 2014), empathic dispositions (Choi et al., 2014) and personality traits, such as antisocial behavioral tendencies (Pfabigan et al., 2012), might affect visual processing of emotional stimuli. Because the rapid perception of negative cues in social environments is highly adaptive, the influence of personality traits on visual processing of emotional stimuli, such as emotional body expressions, is an important avenue for research. One relevant trait is alexithymia, a multifaceted personality construct that is expressed with varying intensity in the general population, and characterized by a deficit in identifying, differentiating and describing feelings (Herbert et al., 2011; Parker et al., 2008; Taylor et al., 1991). Importantly, people with high levels of alexithymia exhibit difficulties not only in processing their own emotions, but also in processing the emotions expressed by others (Parker et al., 1993; Sifneos, 1973). Alexithymic individuals show altered recognition of emotional stimuli (Grynberg et al., 2012; Ihme et al., 2014) and decreased activation of the amygdala during presentation of emotional stimuli (Jongen et al., 2014; Moriguchi and Komaki, 2013), specifically negative stimuli (Kugel et al., 2008; Pouga et al., 2010; Reker et al., 2010; for a recent metaanalysis: van der Velde et al., 2013). However, it is unknown whether early visual processing of the emotional information conveyed by body postures might be similarly affected. Thus, this study was 
designed to investigate, using the high temporal resolution of ERPs, whether participants with low levels of alexithymia (LA) and high levels of alexithymia (HA) show similar electrophysiological modulations in response to body postures conveying information about others' actions and emotions. We studied both the early stage of structural body encoding, indexed by the N190 component, and the later stage of visual selective attention, reflected by the subsequent EPN component. In line with evidence suggesting impaired processing of emotional stimuli in alexithymia (Grynberg et al., 2012; Ihme et al., 2014), we expected that only LA participants would exhibit detailed visual encoding of the emotional content of body postures, with the greatest N190 amplitude in response to fearful body postures. Indeed, we expected that HA participants would not show any emotional modulation at the early stage of structural encoding, and, in particular, no fearrelated enhancement of the N190 component. In addition, we explored whether alexithymia might also influence a later stage of perceptual representation, reflecting selective attention to salient stimuli (EPN).

\section{Methods}

\section{Participants}

Three-hundred university students completed the 20-item Toronto Alexithymia Scale (TAS-20; Taylor et al., 2003). Individuals with high and low TAS-20 total scores $(n=18$, top quartile score $>61 ; \mathrm{n}=16$, bottom quartile score $<36$ ) were selected in order to obtain a sample with as large a variance on alexithymia as possible. The alexithymia module of the structured interview for the Diagnostic Criteria for Psychosomatic Research (DCPR) (Mangelli et al., 2006; Porcelli and Rafanelli, 2010; Porcelli and Sonino, 2007), previously used in alexithymia research (Grandi et al., 2011), was also used in the present study to further confirm the presence or absence of alexithymia. In addition, due to the high association between alexithymia and depression (Allen et al., 2011; Hintikka et al., 2001; Honkalampi et al., 2000), the Beck Depression Inventory (Beck et al., 1961) 
was administered to exclude participants with high levels of depression. Participants were included in the study if $i$ ) they had no history of neurological, major medical or psychiatric disorder and ii) their scores on the TAS-20 and the DCPR were congruent. Two participants with a high TAS-20 score and a low DCPR score were discarded; no participants reported high levels of depression on the BDI. All participants had equivalent educational backgrounds and were students at the University of Bologna. Thirty-two right-handed healthy volunteers were selected to take part in the experiment after screening for alexithymia: 16 HA participants (TAS, mean \pm standard deviation: $63.62 \pm 2.68$; 6 males; mean age 20.68; range 18-25 years old) and 16 LA participants (TAS, mean \pm standard deviation $31.56 \pm 2.75 ; 6$ males; mean age 21.18 ; range $19-26$ years old). The two groups were matched in terms of sex and age. The two groups did not differ in terms of BDI score $(t(30)=-1.41 ; p=.16)$. All participants gave their written informed consent to participate after having been informed about the procedure and the purpose of the study. The study was designed and performed in accordance with the ethical principles of the Declaration of Helsinki, and was approved by the Ethics Committee of the University of Bologna Psychology Department.

\section{Experimental task}

The experimental session was run in a sound-attenuated and dimly lit room. Participants sat in a relaxed position on a comfortable chair in front of a 17', PC monitor (refresh rate: $60 \mathrm{~Hz}$ ) at a distance of approximately $57 \mathrm{~cm}$. Prior to the experiment, a short practice session was administered to familiarize participants with the task.

The stimuli were presented on a PC running Presentation software (Version 0.60; www.neurobs.com), and consisted of 64 color pictures of human bodies ( 2 males and 2 females; $10^{\circ} \mathrm{X} 16^{\circ}$ ) in which faces were blanked out. The images were selected from a validated database (Borgomaneri et al., 2015a, 2015b, 2012). Half of the stimuli were the original pictures, and the other half were mirror-reflected copies. The stimuli represented bodies in different postures, in 
which motion was either absent (static body posture) or implied. The implied motion postures depicted either neutral or emotionally expressive body movements. In particular, the body images included 16 static body postures (static body stimuli), in which neither motion nor emotion was implied, 16 neutral body postures in which motion was implied (neutral body stimuli), 16 fearful body postures in which motion was implied (fearful body stimuli) and 16 happy body postures in which motion was implied (happy body stimuli; Figure 1). Three psychophysical studies using the same body images (Borgomaneri et al., 2015a, 2015b, 2012) provided evidence that the neutral, fearful and happy body stimuli are subjectively rated as conveying the same amount of implied motion, and as conveying more body motion than the static body stimuli. Moreover, the fearful and happy body stimuli were rated as more arousing than the neutral and static body stimuli. Critically, while fearful and happy body stimuli were respectively rated as negative and positive, in terms of valence, these two classes of stimuli received comparable arousal ratings.

The stimuli were displayed against a white background, $11^{\circ}$ to the left (left visual field presentation; LVF) or the right $(\mathrm{RVF})$ of the central fixation point $\left(2^{\circ}\right)$. Each trial started with a central fixation period $(100 \mathrm{~ms})$, followed by the stimulus $(500 \mathrm{~ms})$. Participants were asked to keep their gaze fixed on the central fixation point and decide whether the presented stimulus was emotional (fearful or happy body stimuli) or non-emotional (static or neutral body stimuli) by pressing one of two vertically-arranged buttons on the keyboard. Behavioral responses were recorded during an interval of $2400 \mathrm{~ms}$. Half the participants pressed the upper button with their middle finger to report emotional stimuli and the lower button with their index finger to report non-emotional stimuli, while the remaining half performed the task with the opposite button arrangement. Eye movements were monitored throughout the task with electrooculogram (EOG; see below).

Participants performed twelve blocks in an experimental session of approximately 45 minutes. In half of the blocks the stimuli were presented in the LVF, while in the remaining half they were presented in the RVF. Blocks with LVF and RVF presentation were interleaved, and block sequence was counterbalanced between participants. Each block contained 67 trials presented in a 
random order (16 trials x 4 body stimuli: static, neutral, fearful, happy $=64$ trials +3 practice trials). Each participant completed a total of 804 trials. The analysis was run on a total of 768 trials, i.e., excluding practice trials.

\section{Please insert Figure 1 about here}

\section{EEG recording}

EEG was recorded with $\mathrm{Ag} / \mathrm{AgCl}$ electrodes (Fast'n Easy-Electrodes, Easycap, Herrsching, Germany) from 27 electrode sites (Fp1, F3, F7, FC1, C3, T7, CP1, P3, P7, O1, PO7, Fz, FCz, Cz, $\mathrm{CPz}, \mathrm{Pz}, \mathrm{Fp} 2, \mathrm{~F} 4, \mathrm{~F} 8, \mathrm{FC} 2, \mathrm{C} 4, \mathrm{~T} 8, \mathrm{CP} 2, \mathrm{P} 4, \mathrm{P} 8, \mathrm{O} 2, \mathrm{PO} 8)$ and the right mastoid. The left mastoid was used as reference electrode. The ground electrode was placed on the right cheek. Impedances were kept below $5 \mathrm{k} \Omega$. All electrodes were off-line re-referenced to the average of all electrodes. Vertical and horizontal EOG were recorded from above and below the left eye and from the outer canthi of both eyes. EEG and EOG were recorded with a band-pass of 0.01-100 Hz and amplified by a BrainAmp DC amplifier (Brain Products, Gilching, Germany). The amplified signals were digitized at a sampling rate of $500 \mathrm{~Hz}$, and off-line filtered with a $40 \mathrm{~Hz}$ low-pass filter.

\section{ERP data analysis}

ERP data were analyzed using custom routines in MatLab 7.0.4 (The Mathworks, Natic, MA, USA), as well as EEGLAB 5.03 (Delorme and Makeig, 2004), an open source toolbox for EEG data analysis (EEGLAB toolbox for single-trial EEG data analysis, Swartz Center for Computational Neurosciences, La Jolla, CA; http://www.sccn.ucsd.edu/eeglab). Segments of $200 \mathrm{~ms}$ before and $800 \mathrm{~ms}$ after each stimulus onset were extracted from the continuous EEG. The baseline window 
ran from $-100 \mathrm{~ms}$ to $0 \mathrm{~ms}$ relative to stimulus onset. Epochs with incorrect responses were rejected (LA: 5\% and HA: 7\%, across body stimuli and visual fields of presentation). In addition, epochs contaminated with large artifacts were identified using two methods from the EEGLAB toolbox (Delorme et al., 2007): 1) An epoch was excluded whenever the voltage on an EEG channel exceeded $100 \mu \mathrm{V}$ to remove epochs with large EEG peaks; 2) An epoch was excluded whenever the joint probability of a trial exceeded five standard deviations to remove epochs with improbable data (mean excluded epochs HA: 12.71\%; mean excluded epochs LA: 11.53\%). Remaining blinks and EOG horizontal artifacts were corrected using a multiple adaptive regression method (Automatic Artifact Removal Toolbox Version 1.3; http://www.germangh.com/eeglab_plugin_aar/index.html), based on the Least Mean Squares algorithm (Gratton et al., 1983). Finally, epochs were discarded from the analysis when saccadic movements $(>30 \mu \mathrm{V}$ on horizontal EOG channels) were registered in a time window of $500 \mathrm{~ms}$ following stimulus onset (HA: $0.98 \%$; LA: $2.44 \%$ ). The remaining epochs (HA mean number of epochs per condition: 76; LA mean number of epochs per condition: 79) were averaged separately for each participant and each body stimulus type. The N190 amplitude was quantified as the mean amplitude in a time window of $170-220 \mathrm{~ms}$ post stimulus presentation (Figure 2). Scalp topographies for the N190 component were calculated as mean amplitude in a time window of 170-220 ms post stimulus presentation (Figure $3 \mathrm{~A}$ and $3 \mathrm{~B}$ ).

As shown in the scalp topographies (Figure 3A and 3B), the mean N190 amplitude averaged across body stimuli (fearful, happy, neutral and static body postures) and visual fields (LVF and RVF) reached a maximum negative deflection on electrodes P7 and P8. Therefore, electrodes P7 and P8 were chosen as a region of interest (ROI) in the N190 analyses, in line with previous studies (Stekelenburg and de Gelder, 2004; Thierry et al., 2006). Grand average waveforms for LA and HA participants are shown in Figure 2.

In addition, the EPN was calculated as the mean amplitude in a time window of 290-350 ms poststimulus presentation (Figure 2) at the same electrode locations as the N190 (i.e., electrodes P7 and P8). Both the N190 and the EPN mean amplitudes were analyzed with a mixed-model ANOVA 
with Group (HA and LA participants) as a between-participants factor and Electrode (P8 and P7), Visual field (LVF and RVF) and Body stimulus (fearful, happy, neutral and static) as withinparticipants factors. To compensate for violations of sphericity, Greenhouse-Geisser corrections were applied whenever appropriate (Greenhouse and Geisser, 1959), and corrected $p$ values (but uncorrected degrees of freedom) are reported. Post-hoc comparisons were carried out using the Newman-Keuls test.

\section{Results}

\section{ERP results}

\section{N190}

The ANOVA with Group (LA and HA participants) as a between-participants factor and Electrode (P8 and P7), Visual field (LVF and RVF), and Body stimulus (fearful, happy, neutral and static) as within-participants factors showed no significant main effect of $\operatorname{Group}(F(1,30)=0.86 ; p=.36)$, demonstrating that the N190 amplitude was comparable between LA and HA participants. A significant main effect of Electrode $(F(1,30)=5.74 ; p=.02)$ was found, revealing a larger N190 amplitude over electrode P8 in the right hemisphere $(-2.29 \mu \mathrm{v})$ than over electrode P7 in the left hemisphere $(-1.35 \mu \mathrm{v})$. Moreover, the ANOVA showed a main effect of Body stimulus $F(3,90)=$ 46.14; $p<.0001)$. Post hoc analyses revealed that static body postures elicited a significantly smaller N190 amplitude than implied motion postures $(-0.82 \mu \mathrm{v}$; all $p s<.0002)$. Notably, fearful

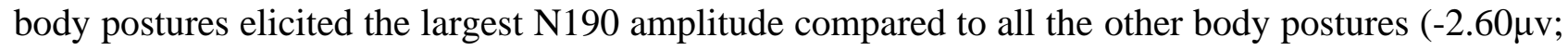
all $p s<.02)$. In addition, neutral body postures $(-2.18 \mu \mathrm{v})$ elicited a significantly larger N190 amplitude than happy postures $(-1.68 \mu \mathrm{v} ; p=.002)$. More importantly, a significant interaction between Body stimulus and Group was found $(F(3,90)=3.70 ; p<.02)$. Post-hoc analyses showed that, in the LA group, static body postures elicited a significantly smaller N190 amplitude (-1.05 $\mu \mathrm{v})$ than neutral body postures $(-2.75 \mu \mathrm{v} ; p=.0001)$, happy body postures $(-1.89 \mu \mathrm{v} ; p=.002)$ and 
fearful body postures $(-3.22 \mu \mathrm{v} ; p=.0001)$. In addition, neutral body postures elicited a larger N190 than happy body postures $(p=.001)$. Importantly, a significantly larger N190 amplitude was found in response to fearful body postures $(-3.22 \mu \mathrm{v})$ compared to both happy $(p=.0001)$ and neutral body postures ( $p=.04$; Figure 3C and 3E). Conversely, in the HA group, the N190 amplitude was only modulated by the presence of implied motion. Indeed, static body postures $(-0.58 \mu \mathrm{v})$ elicited a significantly smaller N190 amplitude than implied motion postures (fearful $=1.97 \mu \mathrm{v}$; happy $=$ $1.47 \mu \mathrm{v} ;$ neutral $=1.62 \mu \mathrm{v} ;$ all $p s<.0006)$. In contrast, there were no significant differences in N190 amplitude between positive and negative emotional body postures (fear vs happy: $p=.13$ ) or between emotional and neutral body postures (fear vs neutral: $p=.27$; happy vs neutral: $p=.51$ ) (Figure 3D and 3F). Interestingly, no other factors interacted with the factor Group (all $p s>.24$ ).

Please insert Figures 2 and 3 about here

\section{EPN}

The ANOVA with Group (LA and HA participants) as a between-participants factor and Electrode (P8 and P7), Visual field (LVF and RVF) and Body stimulus (fearful, happy, neutral and static) as within-participants factors showed no main effect of Group, $(F(1,30)=0.06 ; p=.81)$, suggesting similar EPN amplitudes between LA and HA participants (Figure 4).

There was a significant main effect of Body stimulus $(F(3,90)=13.09 ; p<.0001)$. Post-hoc comparisons showed a more negative amplitude in response to fearful body postures $(1.07 \mu \mathrm{v})$, compared to all other postures (happy $=1.76 \mu \mathrm{v}$; neutral $=2.07 \mu \mathrm{v}$; static $=2.08 \mu \mathrm{v}$; all $p s<.0005$ ). Notably, at variance with the results of the N190, no significant interaction between Body stimulus and Group was found $(F(3,90)=0.08 ; p=.97)$ (Figure 4). No other interactions with the factor Group were significant (Visual Field x Group: $p=.06$; all the remaining interactions: $\mathrm{p}>.27$ ). 


\section{Behavioral results}

Reaction times and accuracy scores were analyzed by means of two separate ANOVAs with Group (LA and HA participants) as a between-participants factor, and Visual field (LVF and RVF) and Body stimulus (fearful, happy, neutral and static) as within-participants factors. The ANOVA on reaction times showed no significant difference between the two groups $(F(1,30)=0.06 ; p=.81)$. Only a significant main effect of Body stimulus $(F(3,90)=73.18 ; p<.0001)$ was found. Post-hoc comparisons showed faster reaction times to static body postures (634ms) than to the other body postures $($ fearful $=753 \mathrm{~ms}$; happy $=748 \mathrm{~ms}$; neutral $=769 \mathrm{~ms}$; all $p s<.0002)$. No other comparisons were significant (all $p s>.11$ ). Similarly, the ANOVA on accuracy scores showed no significant difference between groups $(F(1,30)=2.47 ; p=.13)$. A significant main effect of Body stimulus $(F(3,90)=12.02 ; \mathrm{p}<.0001)$ was revealed. Post-hoc analyses showed that participants were more accurate in responding to static body postures (99\%), compared to all the other postures $($ fearful $=92 \% ;$ happy $=94 \%$; neutral $=92 \% ;$ all $p s<.0003)$. No interactions with the factor Group were significant (all $p s>.18$ ).

\section{Discussion}

Human body postures represent a highly relevant vehicle through which both actions and emotions are expressed. Here we tested the hypothesis that individuals with difficulties in emotional processing (i.e., HA participants) may show an altered emotion-related modulation of the visual processing of body postures. 
Consistent with results from participants in the normal range of alexithymia (Borhani et al., 2015), individuals with low alexithymia scores (LA participants) showed N190 modulation both for implied body motion (i.e., body postures implying motion elicited greater N190 amplitudes than static postures) and for the emotional body expressions (i.e., fearful body postures elicited the largest N190 amplitude). These findings reveal fine perceptual discrimination of both the presence of motion and the emotional content conveyed by body postures at an early stage of visual processing. In contrast, HA participants did not show emotion-related modulations of the N190 component. Indeed, the N190 amplitude was significantly enhanced by implied motion, but no emotion-related modulation was observed. Most importantly, the fearful body postures failed to elicit the largest N190 amplitude. These results suggest that, at variance with LA participants and with participants in the normal range of alexithymia (Borhani et al., 2015), HA participants did not show an early detailed perceptual analysis of the emotional content of body postures. In contrast, early discrimination of motion-related information was observed in both LA and HA groups.

At the later stage of visual processing represented by the EPN component, no significant differences were found between HA and LA participants. A more pronounced EPN component was observed in response to fearful body postures in both groups, suggesting a similar attentional capture driven by salient cues in both HA and LA participants.

The lack of any difference in the N190 amplitude elicited by emotional (fearful and happy) and non-emotional (neutral) body postures in HA participants suggests the presence of an altered early perceptual process in alexithymia. This result is in line with previous studies showing impaired early processing of emotional stimuli in alexithymic individuals. Indeed, HA participants showed a reduction in the amplitude of the early visual P1 component in response to emotional pictures (Pollatos and Gramann, 2011). In addition, altered modulations of the P1 and the N1 component were found in emotional oddball tasks (Campanella et al., 2012; Delle-Vigne et al., 2014). Notably, in the present study, HA participants also failed to show the enhanced visual encoding of fearful 
body postures that was evident in LA participants and individuals in the normal range of alexithymia (Borhani et al., 2015). In keeping, individuals with high alexithymia show altered early responses to other types of negative stimuli, such as early auditory-related potentials in response to potentially dangerous acoustic stimuli (Schäfer et al., 2007), reduced electrodermal responses to negative pictures (Pollatos et al., 2008) and impaired internal somatic simulation of fearful faces (Scarpazza et al., 2014). Similarly, HA participants exhibit hypo-responsiveness to negative emotional stimuli in several cerebral areas, including the fusiform gyrus (Deng et al., 2013; Eichmann et al., 2008; Karlsson et al., 2008), the insula, the superior temporal gyrus and the middle occipital and parahippocampal gyrus (Reker et al., 2010) and in brain areas typically involved in emotional processing, such as the amygdala (Jongen et al., 2014; Kugel et al., 2008; Pouga et al., 2010; see van der Velde et al., 2013 for a recent meta-analysis).

The reported hypo-responsiveness of the amygdala to negative stimuli in HA individuals might be responsible for the lack of N190 modulation by fearful body postures. Fearful bodies have been shown to elicit increased activation in the temporo-occipital areas from which the N190 originates (Grèzes et al., 2007; Hadjikhani and de Gelder, 2003; Peelen et al., 2007; Pichon et al., 2008; van de Riet et al., 2009). In addition, fearful bodies enhance activation in the amygdala (de Gelder et al., 2004; Hadjikhani and de Gelder, 2003; van de Riet et al., 2009), the key subcortical structure for signaling fear and potential threat (Adolphs, 2013; LeDoux, 2014). Therefore, the typical enhancement of the N190 in response to fearful body postures, observed in LA individuals and in those with alexithymia scores in the normal range, might reflect a fast, distant functional influence of the amygdala on interconnected visual cortices (Borgomaneri et al., 2014; Vuilleumier et al., 2004; Wendt et al., 2011). Such a mechanism could be altered in individuals with high alexithymia, in which the reduced amygdala responses to negative stimuli (Jongen et al., 2014; Kugel et al., 2008; Pouga et al., 2010; see van der Velde et al., 2013 for a recent meta-analysis) might weaken feedback projections to the visual areas, preventing fear-related modulation of early perceptual processing. 
Interestingly, the altered visual encoding of body postures observed in HA participants was specifically related to their emotional content, while the encoding of motion-related information was unaltered. Indeed, individuals with alexithymia showed the typical enhancement of structural encoding for implied motion postures compared to static postures, suggesting an intact perceptual representation of bodies performing actions, possibly mediated by a wide fronto-parietal network of sensorimotor regions involved in action observation, planning and execution (Gallese and Sinigaglia, 2011; Gallese et al., 2004; Keysers and Gazzola, 2009; Nishitani et al., 2004).

At a later stage of visual processing, the same fear-related enhancement of the EPN component was found in both HA and LA participants, in line with previous results from participants in the normal range of alexithymia (Borhani et al., 2015). The observed enhancement of the EPN with fearful body postures is consistent with previous evidence showing larger EPN components in response to negatively valenced faces (Bayer and Schacht, 2014; Calvo and Beltrán, 2014; Frühholz et al., 2011; Rellecke et al., 2012; Schupp et al., 2004; Valdés-Conroy et al., 2014). Similar to the findings of the present study, an emotional modulation of the EPN has also been observed in response to negatively valenced hand gestures (Flaisch et al., 2011, 2009). These results suggest that, notwithstanding the altered early visual encoding of emotional body expressions, later visual processing of emotional body postures is intact in HA individuals. EPN has been interpreted as a marker of visual selective attention, which might reflect exogenous attentional capture driven by salient and motivationally relevant emotional stimuli (Olofsson et al., 2008; Schupp et al., 2006). The allocation of attentional resources at this later stage relies on a widespread network of brain regions, including extrastriate visual areas, superior and inferior parietal cortices and medial prefrontal regions (for a review, see Pourtois and Vuilleumier, 2006). Therefore, this later stage of visual processing seems at least partially independent of back projections from the amygdala (Wendt et al., 2011). The involvement of a wide network of high order cortical areas, functionally intact in individuals with high alexithymia, seems to account for the preserved electrophysiological 
marker of selective attention towards salient fearful body postures in HA participants. Interestingly, no differences between LA and HA participants were found at the behavioral level, neither in accuracy scores, nor in reaction times. This intact behavioral performance of individuals with high alexithymia is in keeping with the notion that HA individuals show emotional recognition impairments only when exposure times and response windows are short (Grynberg et al., 2012; Ihme et al., 2014).

Overall, the results of the present study suggest that alexithymia specifically affects the early visual encoding of emotional stimuli, without affecting the early visual encoding of motion information. Moreover, alexithymia does not affect fear-related modulation at a later stage of visual processing, which reflects attentional capture of salient stimuli.

\section{Acknowledgments}

We are grateful to Martin E. Maier for his helpful suggestions about ERP data analysis and to Brianna Beck for her help in editing the manuscript. This work was supported by FARB University of Bologna Grant (Protocol: RFBO 120993 - Visual Awareness Lost and Found) to EL. SB was supported by grant from the Cogito Foundation (R-117/13 and 14-139-R) awarded to A. Avenanti. 


\section{Figure captions}

Figure 1. (A) Graphical representation of the trial structure in the behavioral task. The figure depicts an example trial with fearful body posture stimuli. (B) Example stimuli showing fearful, happy, neutral and static body postures.

Figure 2. Grand-average ERPs averaged across electrodes P7 and P8 and across visual fields (LVF and RVF), elicited by fearful, happy, neutral and static body postures in LA participants (A) and HA participants (B).

Figure 3. N190 component. (A - B) 3D Scalp topographies (left back view, central back view, right back view) of the mean N190 amplitude averaged across body stimuli (fearful, happy, neutral and static body postures) and visual fields (LVF and RVF) in a time window of 170-220 ms in LA participants (A) and HA participants (B). (C - D) Mean N190 amplitude elicited by fearful, happy, neutral and static body postures in LA participants (C) and HA participants (D). Error bars represent standard error of the mean (S.E.M.). (D - E) 3D Scalp topographies of the difference in mean N190 amplitude between fearful and other body stimuli (F-H: fearful - happy; F-N: fearful - neutral; F-S: fearful - static) in a time window of 170-220 ms in LA participants (D) and in HA participants (E).

Figure 4. EPN component. (A - B) Mean EPN amplitude elicited by fearful, happy, neutral and static body postures in LA participants (A) and HA participants (B). Error bars represent standard error of the mean (S.E.M.). (C - D) 3D Scalp topographies of the difference in mean EPN amplitude between fearful and other body stimuli (F-H: fearful - happy; F-N: fearful - neutral; F-S: fearful static) in a time window of 290-350 ms in LA participants (C) and in HA participants (D). 


\section{References}

Adolphs, R., 2013. The biology of fear. Curr. Biol. 23, R79-R93.

Allen, L.B., Qian Lu, Tsao, J.C.I., Hayes, L.P., Zeltzer, L.K., 2011. Depression partially mediates the relationship between alexithymia and somatization in a sample of healthy children. $J$. Health Psychol. 16, 1177-86.

Batty, M., Taylor, M.J., 2003. Early processing of the six basic facial emotional expressions. Cogn. Brain Res. 17, 613-20.

Bayer, M., Schacht, A., 2014. Event-related brain responses to emotional words, pictures, and faces: a cross-domain comparison. Front. Psychol. 5, 1-10.

Beck, A.T., Ward, C.H., Mendelson, M., Mock, J., Erbaugh, J., 1961. An inventory for measuring depression. Arch. Gen. Psychiatry 4, 561-71.

Bentin, S., Allison, T., Puce, A., Perez, E., McCarthy, G., 1996. Electrophysiological studies of face perception in humans. J. Cogn. Neurosci. 8, 551-565.

Borgomaneri, S., Gazzola, V., Avenanti, A., 2012. Motor mapping of implied actions during perception of emotional body language. Brain Stimul. 5, 70-76.

Borgomaneri, S., Gazzola, V., Avenanti, A., 2014. Temporal dynamics of motor cortex excitability during perception of natural emotional scenes. Soc. Cogn. Affect. Neurosci. 9, 1451-1457.

Borgomaneri, S., Gazzola, V., Avenanti, A., 2015a. Transcranial magnetic stimulation reveals two functionally distinct stages of motor cortex involvement during perception of emotional body language. Brain Struct. Funct. 220, 2765-81.

Borgomaneri, S., Vitale, F., Gazzola, V., Avenanti, A., 2015b. Seeing fearful body language rapidly freezes the observer's motor cortex. Cortex 65, 232-245.

Borhani, K., Làdavas, E., Maier, M.E., Avenanti, A., Bertini, C., 2015. Emotional and movementrelated body postures modulate visual processing. Soc. Cogn. Affect. Neurosci. 10, 10921101.

Breiter, H.C., Etcoff, N.L., Whalen, P.J., Kennedy, W.A., Rauch, S.L., Buckner, R.L., Strauss, M.M., Hyman, S.E., Rosen, B.R., 1996. Response and habituation of the human amygdala during visual processing of facial expression. Neuron 17, 875-87.

Calvo, M.G., Beltrán, D., 2014. Brain lateralization of holistic versus analytic processing of emotional facial expressions. Neuroimage 92, 237-247.

Campanella, S., Falbo, L., Rossignol, M., Grynberg, D., Balconi, M., Verbanck, P., Maurage, P., 2012. Sex differences on emotional processing are modulated by subclinical levels of alexithymia and depression: A preliminary assessment using event-related potentials. Psychiatry Res. 197, 145-153.

Carretié, L., Albert, J., López-Martín, S., Tapia, M., 2009. Negative brain: an integrative review on 
the neural processes activated by unpleasant stimuli. Int. J. Psychophysiol. 71, 57-63.

Cecere, R., Bertini, C., Maier, M.E., Làdavas, E., 2014. Unseen fearful faces influence face encoding: evidence from ERPs in hemianopic patients. J. Cogn. Neurosci. 26, 2564-77.

Choi, D., Nishimura, T., Motoi, M., Egashira, Y., Matsumoto, R., Watanuki, S., 2014. Effect of empathy trait on attention to various facial expressions : evidence from N170 and late positive potential ( LPP ). J. Physiol. Anthropol. 33, 1-9.

de Gelder, B., Snyder, J., Greve, D., Gerard, G., Hadjikhani, N., 2004. Fear fosters flight: a mechanism for fear contagion when perceiving emotion expressed by a whole body. Proc. Natl. Acad. Sci. U. S. A. 101, 16701-6.

de Gelder, B., Van den Stock, J., Meeren, H.K.M., Sinke, C.B.A., Kret, M.E., Tamietto, M., 2010. Standing up for the body. Recent progress in uncovering the networks involved in the perception of bodies and bodily expressions. Neurosci. Biobehav. Rev. 34, 513-527.

Delle-Vigne, D., Kornreich, C., Verbanck, P., Campanella, S., 2014. Subclinical alexithymia modulates early audio-visual perceptive and attentional event-related potentials. Front. Hum. Neurosci. 8, 106.

Delorme, A., Makeig, S., 2004. EEGLAB: An open source toolbox for analysis of single-trial EEG dynamics including independent component analysis. J. Neurosci. Methods 134, 9-21.

Delorme, A., Sejnowski, T., Makeig, S., 2007. Enhanced detection of artifacts in EEG data using higher-order statistics and independent component analysis. Neuroimage 34, 1443-1449.

Deng, Y., Ma, X., Tang, Q., 2013. Brain response during visual emotional processing: an fMRI study of alexithymia. Psychiatry Res. 213, 225-9.

Eichmann, M., Kugel, H., Suslow, T., 2008. Difficulty identifying feelings and automatic activation in the fusiform gyrus in response to facial emotion. Percept. Mot. Skills 107, 915-22.

Flaisch, T., Häcker, F., Renner, B., Schupp, H.T., Flaisch, T., Ha, F., 2011. Emotion and the processing of symbolic gestures: An event-related brain potential study. Soc. Cogn. Affect. Neurosci. 6, 109-118.

Flaisch, T., Schupp, H.T., Renner, B., Junghöfer, M., 2009. Neural systems of visual attention responding to emotional gestures. Neuroimage 45, 1339-46.

Fox, E., Lester, V., Russo, R., Bowles, R.J., Pichler, A., Dutton, K., 2000. Facial expressions of emotion: are angry faces detected more efficiently? Cogn. Emot. 14, 61-92.

Frühholz, S., Jellinghaus, A., Herrmann, M., 2011. Time course of implicit processing and explicit processing of emotional faces and emotional words. Biol. Psychol. 87, 265-274.

Fusar-Poli, P., Placentino, A., Carletti, F., Landi, P., Allen, P., Surguladze, S., Benedetti, F., Abbamonte, M., Gasparotti, R., Barale, F., Perez, J., McGuire, P., Politi, P., 2009. Functional atlas of emotional faces processing: a voxel-based meta-analysis of 105 functional magnetic resonance imaging studies. J. Psychiatry Neurosci. 34, 418-32. 
Gallese, V., Keysers, C., Rizzolatti, G., 2004. A unifying view of the basis of social cognition. Trends Cogn. Sci. 8, 396-403.

Gallese, V., Sinigaglia, C., 2011. What is so special about embodied simulation? Trends Cogn. Sci. $15,512-9$.

Grandi, S., Sirri, L., Wise, T.N., Tossani, E., Fava, G.A., 2011. Kellner's emotional inhibition scale: A clinimetric approach to alexithymia research. Psychother. Psychosom. 80, 335-344.

Gratton, G., Coles, M.G., Donchin, E., 1983. A new method for off-line removal of ocular artifact. Electroencephalogr. Clin. Neurophysiol. 55, 468-84.

Greenhouse, S., Geisser, S., 1959. On methods in the analysis of profile data. Psychometrika 24, 95-112.

Grèzes, J., Pichon, S., de Gelder, B., 2007. Perceiving fear in dynamic body expressions. Neuroimage 35, 959-967.

Grynberg, D., Chang, B., Corneille, O., Maurage, P., Vermeulen, N., Berthoz, S., Luminet, O., 2012. Alexithymia and the processing of emotional facial expressions (EFEs): Systematic review, unanswered questions and further perspectives. PLoS One 7, e42429.

Hadjikhani, N., de Gelder, B., 2003. Seeing fearful body expressions activates the fusiform cortex and amygdala. Curr. Biol. 13, 2201-2205.

Halgren, E., Raij, T., Marinkovic, K., Jousmäki, V., Hari, R., 2000. Cognitive response profile of the human fusiform face area as determined by MEG. Cereb. Cortex 10, 69-81.

Hansen, C.H., Hansen, R.D., 1988. Finding the face in the crowd: an anger superiority effect. J. Pers. Soc. Psychol. 54, 917-24.

Herbert, B.M., Herbert, C., Pollatos, O., 2011. On the relationship between interoceptive awareness and alexithymia: Is interoceptive awareness related to emotional awareness? J. Pers. 79, 11491175 .

Hintikka, J., Honkalampi, K., Lehtonen, J., Viinamäki, H., 2001. Are alexithymia and depression distinct or overlapping constructs?: A study in a general population. Compr. Psychiatry 42, 234-239.

Honkalampi, K., Hintikka, J., Tanskanen, A., Lehtonen, J., Viinamäki, H., 2000. Depression is strongly associated with alexithymia in the general population. J. Psychosom. Res. 48, 99-104.

Ihme, K., Sacher, J., Lichev, V., Rosenberg, N., Kugel, H., Rufer, M., Grabe, H.J., Pampel, A., Lepsien, J., Kersting, A., Villringer, A., Lane, R., Suslow, T., 2014. Alexithymic features and the labeling of brief emotional facial expressions - an fMRI study. Neuropsychologia 64, 289299.

Jongen, S., Axmacher, N., Kremers, N.A.W.W., Hoffmann, H., Limbrecht-Ecklundt, K., Traue, H.C., Kessler, H., 2014. An investigation of facial emotion recognition impairments in alexithymia and its neural correlates. Behav. Brain Res. 271, 129-139. 
Karlsson, H., Näätänen, P., Stenman, H., 2008. Cortical activation in alexithymia as a response to emotional stimuli. Br. J. Psychiatry 192, 32-38.

Keysers, C., Gazzola, V., 2009. Expanding the mirror: vicarious activity for actions, emotions, and sensations. Curr. Opin. Neurobiol. 19, 666-71.

Kugel, H., Eichmann, M., Dannlowski, U., Ohrmann, P., Bauer, J., Arolt, V., Heindel, W., Suslow, T., 2008. Alexithymic features and automatic amygdala reactivity to facial emotion. Neurosci. Lett. 435, 40-44.

Lane, R.D., Reiman, E.M., Axelrod, B., Yun, L.S., Holmes, A., Schwartz, G.E., 1998. Neural correlates of levels of emotional awareness. Evidence of an interaction between emotion and attention in the anterior cingulate cortex. J. Cogn. Neurosci. 10, 525-35.

LeDoux, J.E., 2014. Coming to terms with fear. Proc. Natl. Acad. Sci. 11, 2871-2878.

Mangelli, L., Semprini, F., Sirri, L., Fava, G.A., Sonino, N., 2006. Use of the Diagnostic Criteria for Psychosomatic Research (DCPR) in a community sample. Psychosomatics 47, 143-146.

Meaux, E., Roux, S., Batty, M., 2014. Early visual ERPs are influenced by individual emotional skills. Soc. Cogn. Affect. Neurosci. 9, 1089-1098.

Meeren, H.K., de Gelder, B., Ahlfors, S.P., Hamalainen, M.S., Hadjikhani, N., 2013. Different cortical dynamics in face and body perception: an MEG study. PLoS One 8, e71408.

Meeren, H.K.M., van Heijnsbergen, C.C.R.J., de Gelder, B., 2005. Rapid perceptual integration of facial expression and emotional body language. Proc. Natl. Acad. Sci. U. S. A. 102, 16518-23.

Moriguchi, Y., Komaki, G., 2013. Neuroimaging studies of alexithymia: physical, affective, and social perspectives. Biopsychosoc. Med. 7, 8.

Nishitani, N., Avikainen, S., Hari, R., 2004. Abnormal imitation-related cortical activation sequences in Asperger's syndrome. Ann. Neurol. 55, 558-62.

Öhman, A., Lundqvist, D., Esteves, F., 2001. The face in the crowd revisited : a threat advantage with schematic stimuli. J. Pers. Soc. Psychol. 80, 381-396.

Olofsson, J.K., Nordin, S., Sequeira, H., Polich, J., 2008. Affective picture processing: an integrative review of ERP findings. Biol. Psychol. 77, 247-265.

Oya, H., Kawasaki, H., Iii, M.A.H., Adolphs, R., 2002. Electrophysiological responses in the human amygdala discriminate emotion categories of complex visual stimuli. J. Neurosci. 22, 9502-9512.

Parker, J.D., Taylor, G.J., Bagby, R.M., 1993. Alexithymia and the recognition of facial expressions of emotion. Psychother. Psychosom. 59, 197-202.

Parker, J.D.A., Keefer, K. V, Taylor, G.J., Bagby, R.M., 2008. Latent structure of the alexithymia construct: a taxometric investigation. Psychol. Assess. 20, 385-396.

Peelen, M. V, Atkinson, A.P., Andersson, F., Vuilleumier, P., 2007. Emotional modulation of bodyselective visual areas. Soc. Cogn. Affect. Neurosci. 2, 274-83. 
Pegna, A.J., Darque, A., Berrut, C., Khateb, A., 2011. Early ERP modulation for task-irrelevant subliminal faces. Front. Psychol. 2, 1-10.

Pegna, A.J., Landis, T., Khateb, A., 2008. Electrophysiological evidence for early non-conscious processing of fearful facial expressions. Int. J. Psychophysiol. 70, 127-136.

Pfabigan, D.M., Alexopoulos, J., Sailer, U., 2012. Exploring the effects of antisocial personality traits on brain potentials during face processing. PLoS One 7, e50283.

Pichon, S., de Gelder, B., Grezes, J., 2008. Emotional modulation of visual and motor areas by dynamic body expressions of anger. Soc. Neurosci. 3, 199-212.

Pollatos, O., Gramann, K., 2011. Electrophysiological evidence of early processing deficits in alexithymia. Biol. Psychol. 87, 113-121.

Pollatos, O., Schubö, A., Herbert, B.M., Matthias, E., Schandry, R., 2008. Deficits in early emotional reactivity in alexithymia. Psychophysiology 45, 839-846.

Porcelli, P., Rafanelli, C., 2010. Criteria for psychosomatic research (DCPR) in the medical setting. Curr. Psychiatry Rep. 12, 246-254.

Porcelli, P., Sonino, N., 2007. Psychological factors affecting medical conditions: A new classification for DSM-V. Adv. Psychosom. Med. 28, I-X.

Pouga, L., Berthoz, S., De Gelder, B., Grèzes, J., 2010. Individual differences in socioaffective skills influence the neural bases of fear processing: The case of alexithymia. Hum. Brain Mapp. 31, 1469-1481.

Pourtois, G., Grandjean, D., Sander, D., Vuilleumier, P., 2004. Electrophysiological correlates of rapid spatial orienting towards fearful faces. Cereb. Cortex 14, 619-633.

Pourtois, G., Thut, G., Grave de Peralta, R., Michel, C., Vuilleumier, P., 2005. Two electrophysiological stages of spatial orienting towards fearful faces: early temporo-parietal activation preceding gain control in extrastriate visual cortex. Neuroimage 26, 149-63.

Pourtois, G., Vuilleumier, P., 2006. Dynamics of emotional effects on spatial attention in the human visual cortex. Prog. Brain Res. 156, 67-91.

Reker, M., Ohrmann, P., Rauch, A. V., Kugel, H., Bauer, J., Dannlowski, U., Arolt, V., Heindel, W., Suslow, T., 2010. Individual differences in alexithymia and brain response to masked emotion faces. Cortex 46, 658-667.

Rellecke, J., Sommer, W., Schacht, A., 2012. Does processing of emotional facial expressions depend on intention? Time-resolved evidence from event-related brain potentials. Biol. Psychol. 90, 23-32.

Rossion, B., Jacques, C., 2008. Does physical interstimulus variance account for early electrophysiological face sensitive responses in the human brain? Ten lessons on the N170. Neuroimage 39, 1959-79.

Scarpazza, C., di Pellegrino, G., Làdavas, E., 2014. Emotional modulation of touch in alexithymia. 
Emotion 14, 602-10.

Schäfer, R., Schneider, C., Tress, W., Franz, M., 2007. Cortical augmenting in alexithymic subjects after unpleasant acoustic stimulation. J. Psychosom. Res. 63, 357-364.

Schupp, H.T., Flaisch, T., Stockburger, J., Junghöfer, M., 2006. Emotion and attention: eventrelated brain potential studies. Prog. Brain Res. 156, 31-51.

Schupp, H.T., Ohman, A., Junghöfer, M., Weike, A.I., Stockburger, J., Hamm, A.O., 2004. The facilitated processing of threatening faces: an ERP analysis. Emotion 4, 189-200.

Sifneos, P.E., 1973. The prevalence of "alexithymic" characteristics in psychosomatic patients. Psychother. Psychosom. 22, 255-62.

Stekelenburg, J.J., de Gelder, B., 2004. The neural correlates of perceiving human bodies: an ERP study on the body-inversion effect. Neuroreport 15, 9-12.

Taylor, G.J., Bagby, R.M., Parker, J.D., 1991. The alexithymia construct. A potential paradigm for psychosomatic medicine. Psychosomatics 32, 153-64.

Taylor, G.J., Bagby, R.M., Parker, J.D. a, 2003. The 20-Item Toronto Alexithymia Scale: IV. Reliability and factorial validity in different languages and cultures. J. Psychosom. Res. 55, 277-283.

Taylor, J.C., Wiggett, A.J., Downing, P.E., 2010. fMRI - Adaptation studies of viewpoint tuning in the extrastriate and Fusiform Body Areas. J. Neurophysiol. 103, 1467-1477.

Taylor, S.F., Liberzon, I., Koeppe, R.A., 2000. The effect of graded aversive stimuli on limbic and visual activation. Neuropsychologia 38, 1415-25.

Thierry, G., Pegna, A.J., Dodds, C., Roberts, M., Basan, S., Downing, P., 2006. An event-related potential component sensitive to images of the human body. Neuroimage 32, 871-9.

Valdés-Conroy, B., Aguado, L., Fernández-Cahill, M., Romero-Ferreiro, V., Diéguez-Risco, T., 2014. Following the time course of face gender and expression processing: A task-dependent ERP study. Int. J. Psychophysiol. 92, 59-66.

van de Riet, W.A.C., Grèzes, J., de Gelder, B., 2009. Specific and common brain regions involved in the perception of faces and bodies and the representation of their emotional expressions. Soc. Neurosci. 4, 101-120.

van der Velde, J., Servaas, M.N., Goerlich, K.S., Bruggeman, R., Horton, P., Costafreda, S.G., Aleman, A., 2013. Neural correlates of alexithymia: A meta-analysis of emotion processing studies. Neurosci. Biobehav. Rev. 37, 1774-1785.

van Heijnsbergen, C.C.R.J., Meeren, H.K.M., Grèzes, J., de Gelder, B., 2007. Rapid detection of fear in body expressions, an ERP study. Brain Res. 1186, 233-41.

Vuilleumier, P., 2002. Facial expression and selective attention. Curr. Opin. Psychiatry 15, 291300.

Vuilleumier, P., Richardson, M.P., Armony, J.L., Driver, J., Dolan, R.J., 2004. Distant influences of 
amygdala lesion on visual cortical activation during emotional face processing. Nat. Neurosci. 7, 1271-8.

Wendt, J., Weike, A.I., Lotze, M., Hamm, A.O., 2011. The functional connectivity between amygdala and extrastriate visual cortex activity during emotional picture processing depends on stimulus novelty. Biol. Psychol. 86, 203-209. 
Figure 1

A
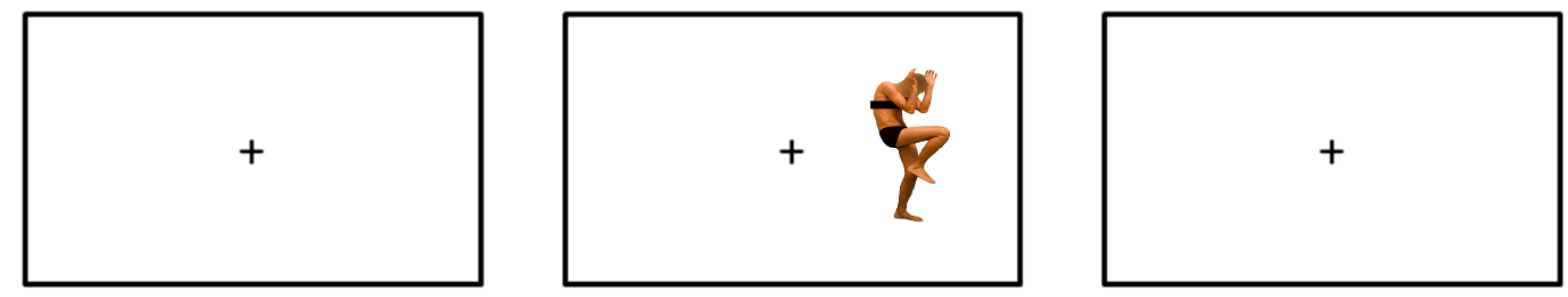

$100 \mathrm{~ms}$

$500 \mathrm{~ms}$

2400 ms / Response Time

B

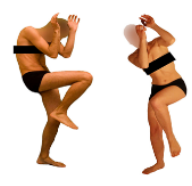

Fearful Body Postures

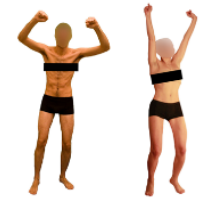

Happy

Body Postures

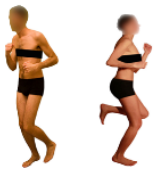

Neutral Body Postures

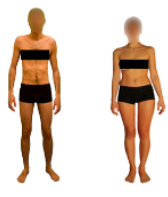

Static

Body Postures 
Figure 2
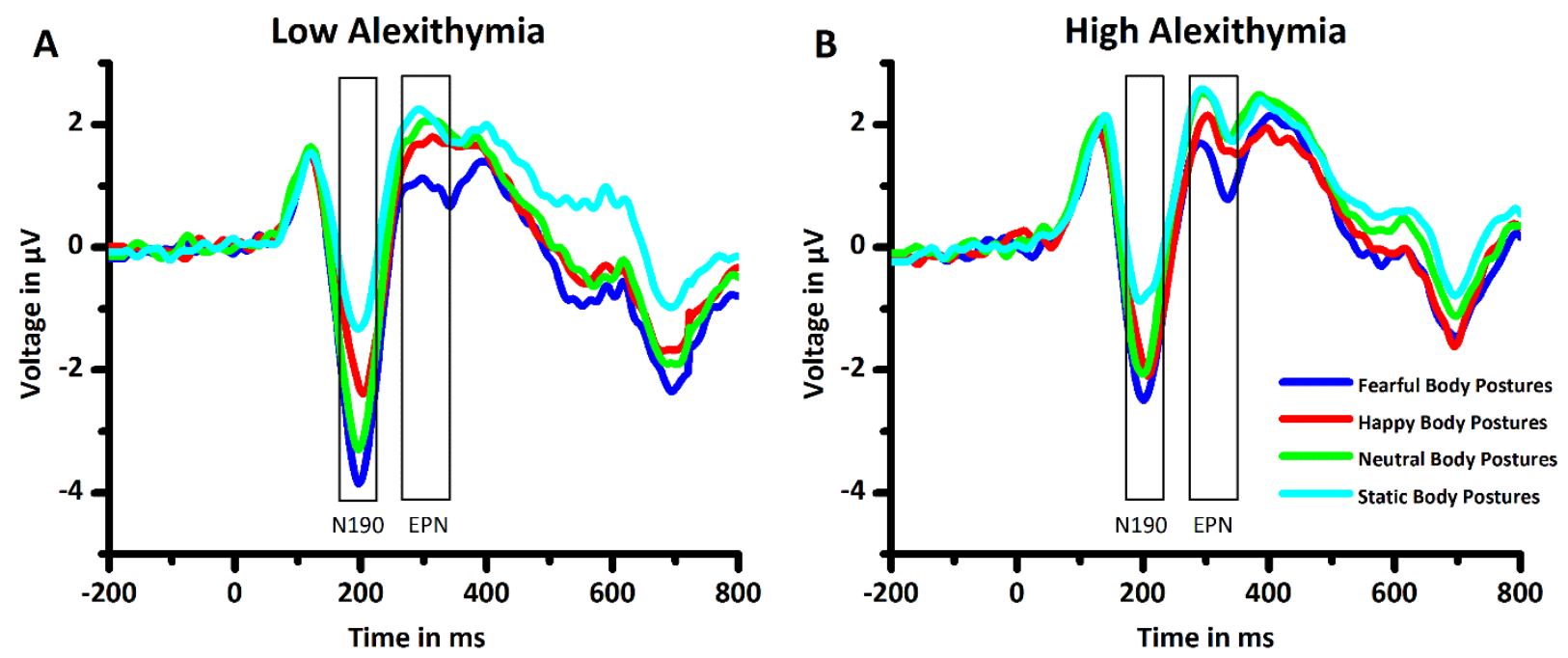
Figure 3

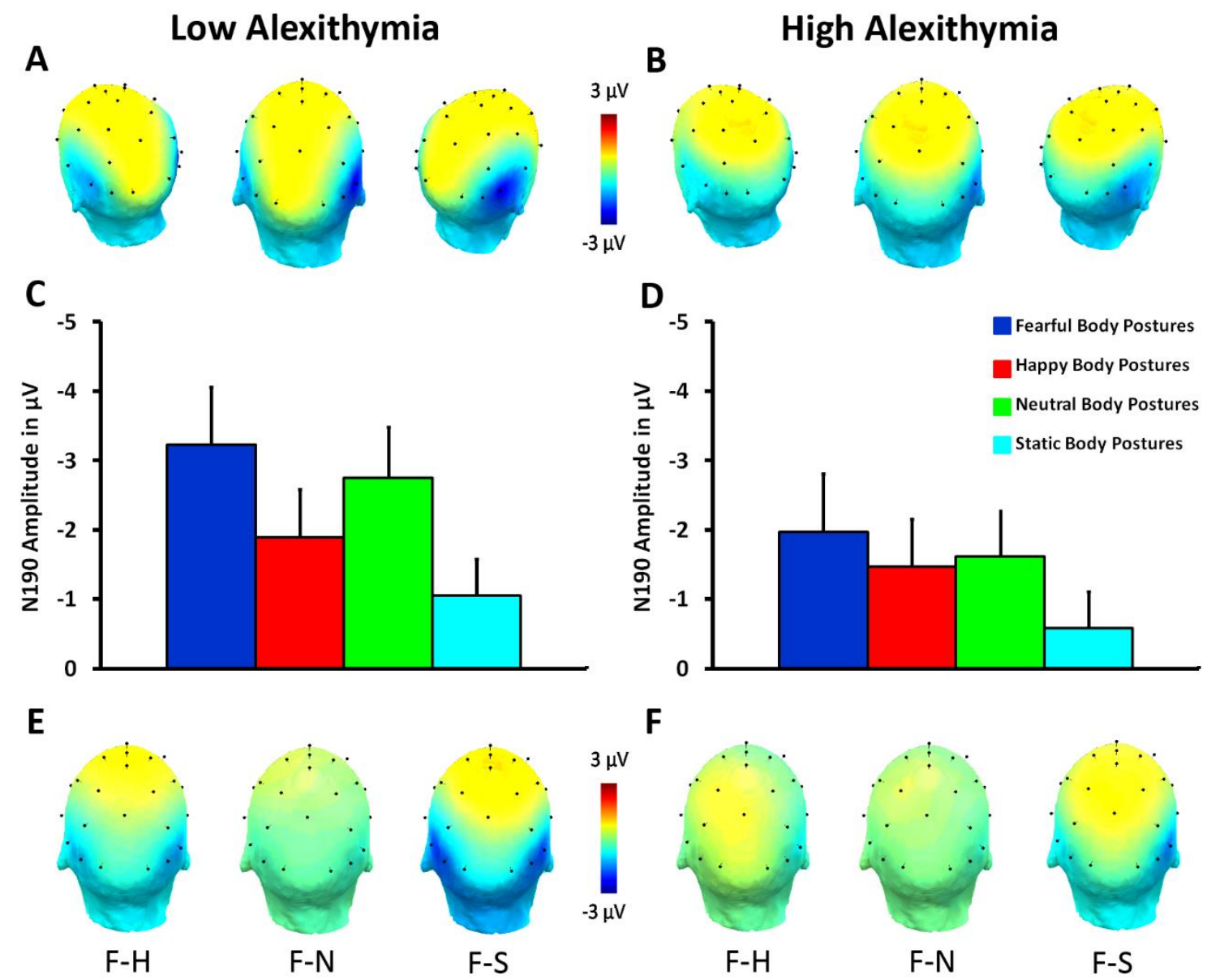


Figure 4

Low Alexithymia

A

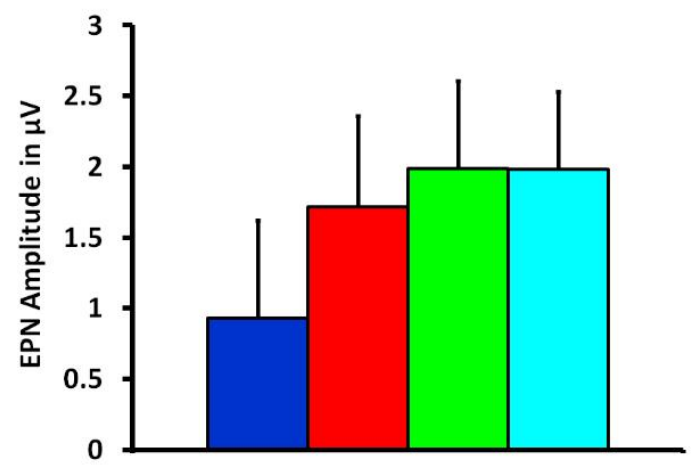

C

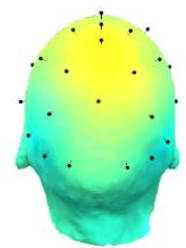

F-H

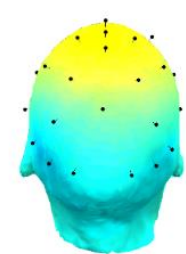

F-N

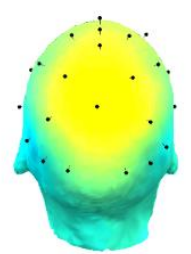

F-S
B

High Alexithymia

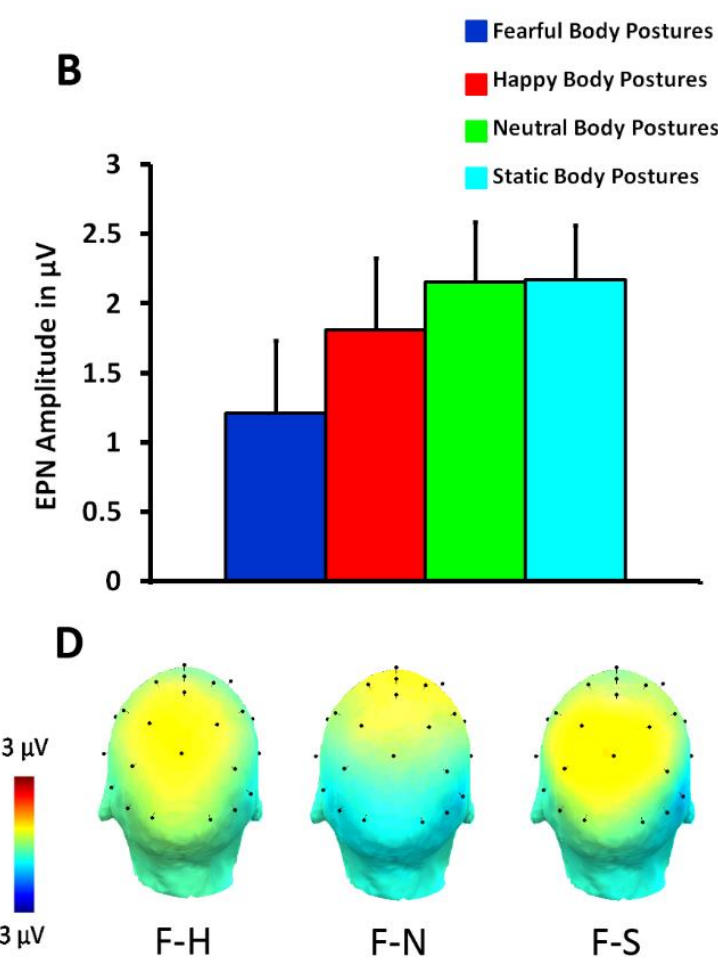

